\title{
Robust Decision Theory and the Lucas Critique
}

Massimiliano Marcellino and Mark Salmon 


\title{
Robust Decision Theory and the Lucas Critique*
}

\author{
Massimiliano Marcellino \\ Università Bocconi \\ Instituto di Economià Politica \\ Mark Salmon \\ City University Business School
}

September 18, 2001

\begin{abstract}
In this paper we re-consider the theoretical basis for the Lucas Critique from the point of view of Robust Decision Theory. We first emphasise that the Lucas Critique rests on a weak theoretical paradigm in that it fails to consider the motivation for the policy change by the government and hence inconsistently assumes limited rationality by the government. When placed in a proper dynamic general equilibrium framework of a dynamic game between the government and the private sector much of the force of the critique simply vanishes. We also re-consider the Critique by adopting an alternative theoretical paradigm and notion of rationality based on robust decision theory. This view of rationality might be regarded as more relevant than the non-robust rationality employed by Lucas and critically it is one in which the Lucas Critique can be shown to simply not apply provided the private sector has adopted suitably robust decision rules.
\end{abstract}

*First Version 1996. This paper incorporates arguments made originally in previously unpublished papers by the two authors; Salmon (1981),(1984) and Marcellino (1995) and represents an abridged version of Marcellino and Salmon (2002). We would like to thank Tom Sargent, Ken Kasa and Ian Petersen for comments on earlier versions and helpful discussions. 


\section{Introduction}

The Lucas Critique, Lucas (1976), is approximately twenty five years old and it may be difficult for some to appreciate the fundamental impact that it had on econometric model building, macroeconomic theory and policy analysis. Given that it denied the possibility of using macroeconometric models for policy simulation and since this was a basic objective of the research programme that started with Tinbergen and Klein it represented a devastating criticism of existing econometric practice.

Over the past twenty five years the economics profession as a whole seems to have adopted a disturbingly unscientific, somewhat schizophrenic pragmatism with regard to the relevance of the critique, driven more by an ideology of convenience than scholarship or empirical evidence. Despite its obvious importance the Critique has received relatively limited theoretical investigation while gaining an enormous citation record ( see Ericsson and Irons (1995)). A number of economists seem to regard the Critique as valid (almost without question) and indeed it has, at least in part, been responsible for stimulating entirely new methodological paradigms such as equilibrium macromodelling (calibration) and the rational expectations econometrics program (see Hansen and Sargent(1980), Sargent(1981)). At the same time many economists simply ignore the Critique and hence by default apparently view it as irrelevant ${ }^{1}$.

Let us quote Lucas at the outset so that we are clear in what follows as to what constitutes the Critique ${ }^{2}$;

"Given that the structure of an econometric model consists of optimal decision rules for economic agents, and that optimal decision rules vary systematically with changes in the structure of series relevant to the decision maker, it follows that any change in policy will systematically alter the structure of econometric models. Lucas(1976, page 41)."

While it is clear therefore that the Critique is an observation on the use of empirical macro-econometric models for policy analysis it is based in theory and on an assumed form of behaviour and the corresponding decision rules which Lucas believes are adopted by economic agents. Hence the Critique may be addressed both on its practical relevance and on its basis in theory.

The Critique has of course been attacked over the years on both theoretical grounds (see in particular, Sims (1980),(1982a),(1982b),(1986),(1987),(1988), 
Cooley et al (1984) and Le Roy (1995)) and empirical grounds ( see Favero and Hendry (1992), Ericsson and Irons(1995) for instance) but the impression still remains that it is an open question as to whether the critique is valid or not and hence how macroeconometric models may be used for policy analysis if at all.

In this paper we re-consider the theoretical justification for the Critique and hence its relevance by reviewing the notion of rational behaviour assumed by Lucas. We have been led to take two separate positions; first we adopt the rationality paradigm Lucas himself uses but extend it to include rational decision making by the policy maker. Since Lucas did not offer any model as to why policy should change and did not attempt to explain the policy maker's behaviour yet regarded the private sector as reactive and rational it seems only natural to impose rationality on the policy maker as well ${ }^{3}$. Secondly we suggest an alternative "rational" paradigm for the development of decision rules which acknowledge the need for a degree of robustness which we believe to be potentially more relevant than the non-robust decision theory employed by Lucas. It is however a notion of rationality in which the Lucas Critique may simply not apply if the private sector has adopted robust rules of behaviour that account for potential variations in government policy.

To state the obvious, it is important to recognise from the outset that the Lucas Critique represents a classic exercise in economic theory and as with all such theoretical propositions in all disciplines it may or may not have any relevance in the real world. Lucas made his critique by imposing on the policymaking process a particular theoretical view of rational behaviour, both for the policymaker and economic agents, and it is this that we believe requires more rigorous theoretical and empirical investigation.

We also need to maintain a clear separation between potentially plausible but purely theoretical constructions and empirical models based on observed behaviour that may bear no relationship whatsoever to the theory. The direct transfer of the implications of any theory to an empirical model by supposition, including the robust paradigm put forward below, is surely poor scientific method and needs to be supported by empirical evidence as to the relevance of the theory: but this is precisely the form of argument used in the Lucas Critique. 


\section{The Lucas Critique; A Statement and Some Observations}

As the quote from Lucas above indicates; the Critique rests on the following syllogism: econometric models represent the behaviour of rational (optimising) agents, who change their behaviour when there are changes in economic policy, hence, when there are changes in policy there are also implied changes in econometric models that seek to capture their behaviour and we cannot use the "old" model to analyse the effects of the "new" policy.

The following characterisation in terms of rational expectations provides a standard description of the critique;

$$
\begin{gathered}
y_{t+1}=\gamma E\left(x_{t+1} \mid I_{t}\right)+\varepsilon_{t} \\
x_{t}=\lambda x_{t-1}+v_{t} \\
y_{t+1}=\vartheta x_{t}+\eta_{t} \quad \text { with } \quad \vartheta=\lambda \gamma
\end{gathered}
$$

Equation (1) is assumed to represent the private sector's optimal decision rule for some variable $y_{t}$ in terms of rational expectations of policy in the next period. This equation together with the policy function of the government, equation (2), is often described as a "structural form" of the model. Equation (3) is then the derived " reduced form" that is assumed to represent the estimated econometric model on which policy simulations would be based. Lucas' argument is that a change in policy, as represented by a new value for instance of $\lambda$, implies a changed value of $\theta$ and hence an econometric model which uses a given estimated value of $\theta$ would not be relevant to explore the value of a new policy which implied a different value of $\lambda$ and hence $\theta^{4}$.

The relevance of the Lucas Critique rests on an understanding of what constitutes a change in policy, why it comes about and whether it necessarily implies a change in behaviour of the private sector that is not already captured by the model. If the private sector's behaviour has anticipated the potential policy changes and this has been properly captured in the econometric model then the Lucas Critique and the potential self conflictory nature of policy simulation stressed by Hendry and Mizon (1996) will not apply. 


\section{$3 \quad$ Rationality and Optimality}

Since Lucas assumes a rational optimising private sector we need, at the outset, to make a relatively simple point which is easily overlooked and that is to be absolutely clear by what is meant by rational behaviour whether it be by the private sector or the government. Some reflection based on standard decision theory leads to the conclusion that rational behaviour, as assumed by Lucas, implies the use of an optimal decision rule which is derived from an explicit specification of all the information needed to solve the initial statement of the intertemporal optimisation problem facing the agent. For instance in a stochastic decision problem this implies that the agents have assumed a knowledge of the stochastic processes that drive the uncertainty they face when they derive their optimal decision rule. Their ignorance of the real world is thus summarised in this stochastic specification and the resulting optimal decision rule is only rational given this specification. Similarly the constraints they face in this optimisation problem define the rationality of the optimal rule at the time when the rule is derived. A rational agent will not knowingly ignore information about his economic environment when forming optimal decision rules.

It is now possible to see a basic theoretical ambiguity and contradiction within the Lucas Critique. Once agents have determined their optimal decision rules there is no need to reconsider them unless the original policy formation failed in some way ${ }^{5}$. However Lucas asks us to consider changes in the optimal rule of the private sector. An optimal rule as we have just stressed, reflects, by assumption, all potential environments that the private agents could imagine they would face including that of government policy. It would be a reflection of irrationality, which Lucas rules out $a b$ initio by explicitly assuming the use of "optimal" decision rules, if the need arose for the private sector to reconsider their original decision rules. As it stands from Lucas' formulation of the policy problem either the optimal decision rule is chosen by the rational private sector in the first place and hence there would be no need to reconsider any change in their decision rule since it would already incorporate an optimal response to changing government policy actions or the original decision rule was not fully optimal which contradicts Lucas' basic initial assumption ${ }^{6}$.

Furthermore, in a "strict" rational expectations paradigm, following Lucas, all agents including the policy maker are supposed to be able to formulate their desires in terms of objective functions, to know the constraints they face 
and to be capable of solving the resulting optimization problem. Notice then that the use of rational expectations also implies that agents employ the true probability distribution as their subjective distributions when forming their expectations - is this the "true " distribution before the assumed policy change or after? Full rationality, in a dynamic general equilibrium context, must surely imply that this distribution includes a specification of how government policy changes and why and hence would be applicable throughout any change in policy if it is indeed the true distribution.

If these rationality propositions are not upheld then the private sector is not in fact forming optimal rules in the first place, as assumed by Lucas, and his theoretical framework collapses. The Lucas Critique rests paradoxically on a restricted specification of the economic environment which serves as the constraint for the private sector's optimisation problem and in fact irrational expectations ${ }^{7}$ and the implied behaviour is thus at best only partial equilibrium in nature and not descriptive of a general equilibrium. This becomes apparent with the assumed policy change, when Lucas assumes agents are forced to reoptimise and derive new decision rules. His initial "optimal rules" for the private sector are, in contradiction, then seen to be suboptimal. In order to properly assess the implications of imposing rational optimising behaviour on the policy invariance of macroeconometric models we need to fully specify the dynamic general equilibrium model including the behaviour of the government and its interaction with the private sector.

These points are fundamental and question the nature of the economic theory that Lucas calls on for support. Perhaps more importantly this discussion also asks us to consider the nature of the decision rules that are used every day by economic agents and hence captured in the observed data and empirical econometric models.

\subsection{What Constitutes a Policy Change?}

We now also need to understand the nature of the policy change that Lucas considers and whether it necessarily induces a change in private sector behaviour. For the time being we overlook the argument made immediately above regarding the invariance of a truely optimal rule and temporarily accept Lucas' partial equilibrium framework since it is in fact possible to demonstrate the failure of the Critique even within this theoretically inappropriate setting.

The macroeconometric models and econometric practice of the time that Lucas was also explicitly criticising did not, in general, assume endogenous 
policy reaction functions but made a modelling assumption that, at the estimation stage, treated policy variables as "exogenous". Hence simulation exercises which involved a selection of new values for these variables were used to evaluate the effects of different policies on the model. We can think of three forms of policy change relative to a given econometric model; a change in the values taken by a policy variable from those in the estimation period, changing the parameters of an equation that describes a policy rule or reaction function captured in the model or introducing a completely new policy regime either by a new rule or instrument that is not present in the model.

Notice that the Lucas Critique trivially does not apply in the last and most dramatic case of a policy change simply because the situation would in fact never arise. It is after all, impossible to simulate the effects of changing a policy variable that does not appear in the model. Everyone would recognise that econometric policy evaluation would be worthless in this case.

Lucas' view of what constitutes a policy change seems to be that economic agents will necessarily treat changed values for the policy variables as arising from a changed government policy rule ( although invariably unseen and often unannounced ) which they had recognised as an explicit constraint when forming their original decisions ${ }^{8}$. The claim is then that changing a constraint's form through a change in policy will change the structure of the agents' optimal decision rules invalidating the econometric model for policy analysis. Both parts of this supposition as to the nature and effect of a policy change can be questioned on theoretical grounds.

The first part turns on the question of whether the private sector treats the government's actions as given as in an open loop dynamic Nash game (i.e. conditions on a sequence $\left\{x_{t}\right\}_{1}^{\infty}$ ) or whether they take the government's policy rule as given, as in a feedback Nash game (i.e conditions on equation (2)). Clearly if the private sector forms open loop decision rules ( or sequential open loop or adaptive decision rules) then by definition they treat the stochastic process generating the values of the government's policy variables as super-exogenous for the parameters of their own decision rules and the Lucas Critique will not apply ${ }^{9}$. Taking the values of the policy variables as given in effect assumes that the private sector cannot or does not need to learn the form of the implicit policy rule ${ }^{10}$. At a deeper theoretical level it may simply be that an atomistic private sector does not play a strategic game with the policy maker at all and each individual agent simply adopts a passive role treating the government's policy actions as an exogenous sto- 
chastic process and an input into their optimal feedback decision rules. The standard use of the fictional representative agent to represent the private sector and to support models of strategic interaction is theoretically flawed as demonstrated by Kirman(1992) and until this issue of establishing rigorous theoretical ( not necessarily micro) foundations for aggregate behaviour ${ }^{11} \mathrm{can}$ be convincingly resolved it would seem to be impossible to argue one way or the other between the use of (sequential) open loop or feedback decision rules by the private sector theoretically. Nevertheless it is clear that Lucas' presumption that policy variables will not be super exogeneous is not the only theoretical position that could be taken even in a strategic game ${ }^{12}$.

Turning to the second part of the supposition (and ignoring the ambiguity over the first part), we need to address the question of how the private sector would act if it were faced with uncertainty about the relevant constraints including the government's policy rules (or actions) when forming its rational decision rules. One approach would be to formalise the constraints as inexact (through $v_{t}$ in equation (2)) and employ the methods of stochastic decision theory - the route which is standard throughout economics ${ }^{13}$. Sims has frequently made this point when discussing the Lucas Critique and has emphasised that it would only be rational for the private sector to incorporate a stochastic specification for government policy whose range space was sufficiently broad to incorporate any reasonable policy change. Given this specification the appropriate reaction to potential future policies would then be considered within the design of the optimal decision rule at the outset and hence there would be no need to derive a new decision rule simply because a new drawing of the random policy variable had been obtained ${ }^{14}$.

\section{Robust Decision Rules}

This argument to treat the private sector's views as to the potential range of policy changes stochastically returns us to the earlier discussion of what constitutes rational behaviour and hence what is an optimal decision rule. It is now critically important to recognise that by simply adopting a stochastic approach to uncertainty and using a stochastic decision theory does not guarantee robustness in the sense of performance invariance in the face of misspecifications in the assumed constraints or disturbances. A much more powerful approach to dealing with an uncertain environment, which has not yet generally been appreciated as a good basis for rational economic 
behaviour but has been used by control engineers for a number of years is to explicitly employ robust decision theory ${ }^{15}$. Critically this robust decision theory largely treats uncertainty as the existence of deterministic shocks and not stochastic. Hence using robust decision theory we can still maintain Lucas' position of viewing a policy change as a parametric or structural change in a rule (i.e. a change in $\lambda$ in (2)), be it either a deterministic or a stochastic rule, and yet show that the private sector's optimal decision rule will not be required to change with a change in policy. This runs in direct contradiction to Lucas' basic argument. The distinction between the two approaches to forming decision rules under ignorance as to the constraints ( and other aspects of the decision problem), either stochastic or robust decision theory can be seen to be an aspect of the difference between risk and uncertainty as identified by Knight (1921). Robust decision theory has recently emphasised a deterministic approach to modelling the unstructured shocks hitting the decision problem ( Uncertainty) whereas standard stochastic decision theory has employed probability models (Risk). The robust approach essentially does not presume the ability of economic agents to employ probability distributions but argues that they would treat shocks on potentially an individual basis and employ decision rules that would be able to achieve their ultimate objective for a given class of such deterministic shocks.

What we now question is the notion of optimality and rationality that Lucas employs to characterise economic behaviour under uncertainty. Recognising that rationality is only defined relative to a given loss function we can see that instead of using the standard theoretical approach in economics through utility maximisation which describes how economists believed economic agents should act we might also wish to consider robust behavioural motivations that might better describe how people actually do behave. Rosenbrock and McMorran (1971), distinguish for instance between Good, Bad and Optimal decision rules; the point is simple; Optimal rules are often not Good rules in the sense that optimality follows from an exact specification of the optimisation problem including the constraints faced. Any deviation from the assumed form of the constraints may yield extremely poor performance from the ex ante "optimal rule". This poor performance in the optimal rule is exactly what leads Lucas to claim that agents' decision rules will change in the face of deviation from the assumed form for the government's policy rules calling on - " everything we know about dynamic economic theory indicates that this presumption ( that the decision rule will not change) is unjustified". We beg to differ, under certain conditions, either assuming a different 
formulation for the loss function employed by economic agents or different state information would lead to optimal but robust decision rules, perhaps as we shall see below with integral action, that would remain invariant in the face of disturbances and shifts in the constraints, such as induced by policy changes whilst still achieving desired performance objectives such as a zero steady state error in tracking some desired equilibrium poistion.

\subsection{Robustness and Integral Action}

It has been standard practice for control engineers to design controllers that achieve their objective given an inexact model of the system to be controlled and hence to allow deviations from the assumed constraints. One simple step in this direction is to suggest that agents formally use PID (Proportional, Integral and Derivative) decision rules which by incorporating integral action recognise the error between current behaviour and some desired position and adjust actions accordingly to ensure that the error is asymptotically zero see Phillips(1954). Salmon (1982), following standard control theory, showed how such PID rules could be derived from within classic intertemporal optimisation problems and led to econometric specifications which have become well known as Error Correction Mechanisms (ECM's) ( see for instance, Hendry and Anderson (1977), Hendry and von UngernSternberg(1981), Nickell(1985) and Hendry (1995) for a recent discussion). Salmon and Young (1978) had in fact earlier demonstrated the ability of such PID rules to achieve a zero steady state error precisely in the case of a parameter change in an equation of an econometric model within a policy optimisation exercise. The point is then that contrary to Lucas' claim it is possible to consider that the private sector use optimal (PID) decision rules that have an invariant structure which is unaffected by perturbations in the assumed constraints but robustly delivers an optimal response in the face of changing government policy of the form considered by Lucas ${ }^{16}$.

Following Athans(1971) we can demonstrate this invariance argument and the need for integral action in robust decision rules with a simple example. Consider a decision maker faced with a constraint described by the following first order system;

$$
\dot{y}(t)=-\alpha_{0} y(t)+\beta_{0} v(t)
$$

This constraint could represent the reduced form of the economic system he

faces including the government's policy reaction function; so a change in the 
parameters of (4) could represent a change in the government's policy rule. His decision variable is $v(t)$ and his objective is to drive $y(t)$ towards some desired position , $y^{*}$, ( a requirement we can express as $\lim _{t \rightarrow \infty} y(t)=y^{*}$ ) which may for instance be the equilibrium described by some classical micro theory. The problem our representative agent faces is to find a practical decision rule recognising his uncertainty about the parameters $\alpha_{0}$ and $\beta_{0}$. Let us define the error $e(t)=y^{*}-y(t)$ then the error dynamics implied by the system are given by;

$$
\dot{e}(t)=-\alpha_{0} e(t)-\beta_{0} v(t)+\alpha_{0} y^{*}
$$

A standard approach would then be to set up the optimisation problem;

$$
\min _{v(t)} \int_{0}^{\infty}\left[g e^{2}(t)+v^{2}(t)\right] d t
$$

Without discounting there is no solution to this problem as the convergence of the cost integral requires $\lim _{t \rightarrow \infty} e(t)=0$ and $\lim _{t \rightarrow \infty} v(t)=0$ but the optimal solution implies $\lim _{t \rightarrow \infty} v(t)=\frac{\alpha_{0}}{\beta_{0}} y^{*} \neq 0$. More importantly for the present discussion the dependence of the optimal decision variable on the assumed values of $\alpha_{0}$ and $\beta_{0}$ is also clear and if the true constraint were to be given by different values for these parameters, say $\alpha$ and $\beta$ then the desired target would not be achieved. Given in this case that the target is a constant there would be a constant steady state error given by,

$$
e_{s s}(t)=\left(1-\frac{\beta \alpha_{0}}{\alpha \beta_{0}}\right) y^{*}
$$

One way to introduce integral action into economic agents' decision rules which then ensures the specified target behaviour will be achieved independently of the specific values taken by the constraint parameters is to allow the rate of change of the decision variable to enter into the cost function. Let us define $u(t)$ as $\dot{v}(t)$. Then we can set up the state space model and optimisation problem as follows; let the state variables be defined as, $x_{1}(t)=e(t)$ and $x_{2}(t)=\dot{e}(t)$ so that

$$
\begin{gathered}
\dot{x}_{1}(t)=x_{2}(t) \\
\dot{x}_{2}(t)=-\alpha_{0} x_{2}(t)-\beta_{0} u(t)
\end{gathered}
$$

then by optimising

$$
\int_{0}^{\infty}\left[q x_{1}^{2}(t)+u^{2}(t)\right] d t
$$


subject to the state space model (7)we get by standard linear quadratic optimisation theory that

$$
u(t)=g_{1} x_{1}(t)+g_{2} x_{2}(t)
$$

where

$$
\begin{gathered}
g_{1}=\sqrt{q} \\
g_{2}=\frac{1}{\beta_{0}}\left[-\alpha_{0}+\sqrt{\left(\alpha_{0}^{2}+2 \beta_{0} \sqrt{q}\right)}\right]
\end{gathered}
$$

Hence the optimal decision rule is given by

$$
v(t)=g_{2} e(t)+g_{1} \int e(\tau) d \tau
$$

which has proportional and integral terms acting on the "disequilibrium error", $e(t)$. Notice however that this decision rule ensures a zero steady state error for any simple parameter changes as can be seen since the closed loop gain is unity in the limit or alternatively by applying the final value theorem of Laplace transforms to the error dynamics which are given by,

$$
\ddot{e}(t)=-\alpha_{0} \dot{e}(t)-\beta_{0} \dot{v}(t)=-\left(\alpha+\beta g_{2}\right) \dot{e}(t)-\beta g_{1} e(t)
$$

we can see that $\lim _{t \rightarrow \infty} e(t)=0$ for any value of $\alpha, \beta>0$ and $y^{*}$ which ensure stability of the error dynamics. The critical insight is that by introducing the derivative of control action into the cost function we ensure that $\lim _{t \rightarrow \infty} \dot{v}(t)=0$ which is equivalent to ensuring that $\lim _{t \rightarrow \infty} v(t)=c$ where $c$ is any unknown constant $^{17}$.

There are several different ways of introducing integral action into agents' decision rules( see virtually any control text) and indeed many naturally occurring economic variables may induce integral action and through a proper specification of stock and flow equilibrium economic behaviour may naturally ensure a zero steady state error without any particular need to respecify the agents' loss function as suggested by the above example. Notice also, as discussed in Salmon (1982), that simple integral action may not be sufficient in the face of more general changes in the constraint structure than simple parameter shifts. The most general theory developed in the control literature following this approach for this disturbance rejection and tracking problem implies, as described in Salmon (1988), that the economic agent should form 
an "internal model" ( see Francis and Wonham(1976)) of the government's behaviour which is then formally exploited in the design of its invariant decision rule which would then be able to achieve the desired optimal economic objective regardless of any changes in government actions. However this is no more and no less than the assumption that Lucas himself uses when he assumes that economic agents employ rational expectations or in the dynamic general equilibrium implied by a dynamic game. The subjective model of the true environment which economic agents use to form their rational expectations and their decision rules must include this internal model and then the internal model principle implies that they will therefore be able to construct invariant robust decision rules in the face of changing government behaviour. Dynamic general equilibrium rational macro theory would then in fact seem to deny the Lucas Critique ${ }^{18}$.

\subsection{Robust Decision Rules and $H^{\infty}$}

Robust control theory has recently developed along a number of different lines, see Zhou, Doyle and Glover (1996) and Petersen et al (2000). The most significant advance in robust decision theory has been the development of $H^{\infty}$ theory (see Basar and Bernhard (1995) or Green and Limebeer(1995)) which has considerably relaxed the nature of the lack of knowledge regarding the environment facing the decision maker enabling robust rules in the face of norm bounded deviations from a nominal model. It is important to recognise however that the standard economist theorist's route of adopting a statistical framework for decision making under uncertainty is not normally employed today by practical control engineers seeking robust control rules. $H^{\infty}$ theory was developed within a deterministic frequency domain environment and can be seen as an extension of decision making under Knightian uncertainty in which a worst case scenario is conceived and the best decision in the face of this potential worst case then computed. The disturbances may in fact have no stochastic interpretation at all and may be purely deterministic once off shocks. The result is a maxmin strategy which is common in other areas of robust analysis such as statistics where the objective is to ensure that disturbances have as little effect as possible on the output.

The environment we envisage the rational economic agent as inhabiting is dynamic and hence there is a dynamic mapping from the shocks to the output variables which is captured by a suitably defined transfer function or state space form for the nominal model. Rationality now implies the need to 
minimise the impact of mispecifications, or shocks so that the agents decision rules satisfy given performance bounds for all disturbances within some norm bounded set. Structured or unstructured disturbances to this nominal model can take a wide variety of forms, either as additive or multiplicative mispecifications in terms of parameters or quite general frequency dependent disturbances bounded within some region of the nominal model. $H^{\infty}$ theory has established several alternative routes to obtaining linear feedback decision rules that provide the desired performance in the face of arbitrary misspecifications satisfying the norm bounded constraints. Basar and Bernhard(1995) provide a dynamic game interpretation of $H^{\infty}$ decision theory in which nature, as the other player, attempts to construct the worst case strategy that you could face and then you construct the best decision rule you can given that Nash assumption. The saddle point structure that results from this game provides a min-max solution to the robust policy design problem. Whittle (1990),(1996) has discussed the relationship between risk sensitive decision theory and $H^{\infty}$ theory and shows how the stochastic formulation of risk sensitivity can lead to an equivalent decision rule as that from $H^{\infty}$ which is in principle deterministic. So a demand for robustness to deterministic shocks to utility can be equivalent to risk sensitive behaviour. Hansen, Sargent and Tallarini (2000), also discuss how this demand for robustness ties in with Epstein-Zin (1989) recursive utility theory and Gilboa-Shmeidler's (1989) version of Knightian uncertainty.

In the context of the Lucas Critique we consider the case of economic agents face uncertainty in the policy rule of the government and hence employing $H^{\infty}$ decision rules in response which incorporate in their potential range of uncertainty all rules the government may adopt. The argument here is simply that they form the best response decision rule given a prespecified range of alternative rules that could describe the government's actions. Given a reference model $M$, they seek, in the terminology of Onatski and Stock(2002), to minimise the risk $R(K, M+\Delta)$ associated with employing decision rule $K$ given a potential range of perturbed models , $M+\Delta$, where $\Delta \in D$, corresponding to different government policy rules. The robust decison problem facing the private sector agent then corresponds to solving

$$
\min _{\{K\}} \sup _{\Delta \in D} R(K, M+\Delta)
$$

where the range of alternative policies which could be delivered by the government could extend as far as the worst possible the agent could face. The 
result is a fixed coefficient feedback rule that is designed to provide good behaviour over a range of alternative constraints rather than be optimal for just one. The later is of course the non-robust case considered by Lucas in the Critique.

In a general dynamic setting we could consider the private sector constructing decision rules in the face of both additive (different government policy rules) and multiplicative ( eg. parameter or non-stochastic) uncertainty. As a simple example we will assume a state space form for the constraints the private sector face which includes the government's decision rules as additive adjustments to a nominal model as in Onatski and Stock above,

$$
\begin{aligned}
& x(t)=A x(t)+B_{u} u(t)+B_{w} w(t) \\
& y(t)=C x(t)+D u(t)
\end{aligned}
$$

where the private sector wants to determine its optimal rule for the decision variable $u$ and $w$ represents disturbances or the arbitrarily specified alternative forms of government policy expressed as deviations from the nominal model, $y$ represent the observable output variables. A number of different formulations of the form of the uncertainty could be made. Notice the state variable $x(t)$ may include both backward looking and forward looking variables such as asset prices as in Miller and Salmon (1985a) and hence may also include forward looking expectational terms.

The $H^{\infty}$ decision problem then seeks to find the feedback rule that minimises the closed loop $H^{\infty}$ norm or alternatively a quadratic objective function can be constructed by considering the bound on the closed loop $\infty$ norm, representing the linear operator or transfer function of the disturbances to the output variables,

$$
\left\|G_{y w}\right\|_{\infty}=\sup _{\|w(t)\|_{2} \neq 0} \frac{\|y(t)\|_{2}}{\|w(t)\|_{2}}<\gamma
$$

where $\gamma$ is called the performance bound. Notice that $\gamma$ is inversely related to the norm bound on the uncertain inputs $\|w(t)\|_{2}$. A decision rule satisfying this will also satisfy the squared bound so

$$
\left\|G_{y w}\right\|_{\infty}^{2}=\sup _{\|w(t)\|_{2} \neq 0}\left\{\frac{\|y(t)\|_{2}^{2}}{\|w(t)\|_{2}^{2}}\right\}<\gamma^{2}
$$

and for the supremum to satisfy the strict inequality the term within the 
brackets must be bounded away from $\gamma^{2}$ so that for some $\varepsilon$

$$
\frac{\|y(t)\|_{2}^{2}}{\|w(t)\|_{2}^{2}} \leq \gamma^{2}-\varepsilon^{2}
$$

and then multiplying through by the denominator yields

$$
\|y(t)\|_{2}^{2}-\gamma^{2}\|w(t)\|_{2}^{2} \leq-\varepsilon^{2}\|w(t)\|_{2}^{2}
$$

The satisfaction of this inequality for all disturbances and some $\varepsilon$ is equivalent to the bound on the closed loop $\infty$ norm and the left hand side of this expression can be used as an objective function.

$$
J_{\gamma}(x, u, w)=\|y(t)\|_{2}^{2}-\gamma^{2}\|w(t)\|_{2}^{2}
$$

Standard tools of differential game theory can now be applied to find the decision rule that that minimises this objective function in the presence of the worst possible disturbance. This leads to a guaranteed performance given by the upper value of the dynamic game,

$$
\inf _{u} \sup _{w} J_{\gamma}(x, u, w)
$$

If we let $\gamma^{*} \equiv \inf \{\gamma:$ the uppervalue in (11) is finite $\}$ then $\gamma^{*}$ is the minimum value of $\left\|G_{y w}\right\|_{\infty}$ that can be obtained. So

$$
\gamma^{*}=\inf _{u}\left\|G_{y w}\right\|_{\infty}
$$

and the decision rule that obtains this infinum is known as the $H^{\infty}$ optimal rule. However in many cases it is sufficient to design as decision rule that corresponds to a suboptimal case which guarantees $\left\|G_{y w}\right\|_{\infty}<\gamma$ with $\gamma>\gamma^{*}$. This can be achieved by approaching as closely as desired on the optimal value $\inf _{u}\left\|G_{y w}\right\|_{\infty}$ iterating on $\gamma$.This suboptimal controller attains the uppervalue for the game corresponding to $\gamma<\gamma^{*}$. Whittle and Bernhard have discussed, in their contributions to this special issue, the relationship between the parametrisation of the stochastic risk sensitivity formulation (LEQG) of the decision problem and its relationship to $\gamma^{*}$ in this deterministic $H^{\infty}$ problem, see also Dupuis et al (2000).

Since the dynamic game above is non-stochastic standard open loop Pontryagin methods can be used to determine the solution with the first order 
conditions or Hamiltonian system obtained from an unconstrained minmax problem with objective function defined using the co-state variables $p$,

$$
J_{\gamma}^{*}(u, w, p)=\int_{0}^{\infty} y^{\prime} y-\gamma^{2} w^{\prime} w+2 p^{\prime}\left(A x+B_{u} u+B_{w} w-\dot{x}\right) d t
$$

The resulting Hamiltonian system is given by

$$
\left[\begin{array}{c}
\dot{x}(t) \\
\dot{p}(t)
\end{array}\right]=\left[\begin{array}{cc}
A & -B_{u} B_{u}^{\prime}+\gamma^{-2} B_{w} B_{w}^{\prime} \\
-C^{\prime} C & -A^{\prime}
\end{array}\right]\left[\begin{array}{c}
x(t) \\
p(t)
\end{array}\right]
$$

with a resulting decision rule given by

$$
u(t)=-B_{u}^{\prime} p(t)
$$

The uniqueness of the robust decision rule for the range of different models defined by the uncertainty bound arises from the uniqueness of the costate process, $p(t)$, which can be obtained either through an eignevector decomposition and the solution to the two point boundary value problem as in Miller and Salmon (1985a) or through the solution to the implied Riccati equation. Notice the generally provided by this Hamiltonian formulation of the $H^{\infty}$ problem. The state vector may be set up so as to include a variety of strategic dynamic games as in Miller and Salmon (1985b) including expectational or forward looking variables ( such as costate variables in the solution of strategically asymmetric or Stackleberg games) and the solution then obtained as a single consistent mapping of the unstable variables onto the stable variables under control, or onto the stable manifold. This single mapping through the solution to the Riccati matrix ensures that policy is consistent with expectations and expectations consistent with policy.

Formally a specification of the norm bounded uncertainty is equivalent via the small gain theorem to solving a (state feedback) $H^{\infty}$ problem for the nominal system and this $H^{\infty}$ problem is in turn equivalent to a particular dynamic game problem with a corresponding Riccati solution. The initial specification of the norm bounded uncertainty set describing the private sector's uncertainty as the government's decision rule translates into a unique costate process for this set of deviations from the nominal model and thus a single robust decision rule can be used by the private sector which satisfies the desired robust performance criteria regardless of changes in the government's policy function within this set. Clearly if the Government chose a policy that 
was not incorporated within the norm bounded uncertainty set considered by the private sector initially then the Lucas Critique would continue to apply. The question of whether or not the Lucas Critique then applies in practice becomes a question of whether or not we believe rational economic agents adopt sufficient robustness within their decision rules.

This very rapid description of the $H^{\infty}$ approach has highlighted the main issue that robust rules may remain invariant within a set of predefined norm bounded uncertainty but we should also note that $H^{\infty}$ rules in common with $H^{2}$ do not naturally incorporate integral action although as with $H^{2}$ decision theory integral effects can be included by construction, See Zhou, Doyle and Glover(1996), section 17.4. Integral action along with the internal model and $H^{\infty}$ decision rules may provide a better behavioural basis for economic theorists to characterise rational economic behaviour than the "knife edge", non-robust utility maximers envisaged in the Lucas Critique. Fundamentally in this case the force of the Critique is critically weakened and may vanish completely. This analysis can be extended to consider economic agents using $H^{\infty}$ decision rules in strategic settings and also to form robust expectations using $H^{\infty}$ filtering methods.

\subsection{Conclusions}

The Lucas Critique raised a range of fundamental issues, both of economic theory and of econometric practice at that time. It emphasised the endogeneity of policy and expectations. At a very basic level it asked the question of what do we mean by rational economic behaviour and hence what are the nature of the empirical relations captured in macroeconometric models. We feel that the Critique failed to recognise the implications of the dynamic general equilibrium that exists between the government and the private sector and hence misinterpreted the nature of agents decision rules and the information within the data sets on which the empirical models mayt be built. If the deep strategic relationship between the government and the private sector were to change then the Critique would have more force but that would require a rational explanation within a dynamic game that we find hard to accept. By failing to recognise the endogeneity of the policy change the critique is logically internally inconsistent in its assumption of rational behaviour and hence the adoption of optimal decision rules both before and after the assumed policy change.

Moreover the form of rational behaviour under uncertainty embodied 
within the Lucas Critique presumes that both parties in the dynamic game do not adopt robust decision rules. We have stressed that if the notion of rationality is extended so that economic agents are seen to employ robust decision rules then these can remain invariant in the face of an wide class of potential changes in government policy and the Critique fails. 


\section{NOTES:}

1. Often by default we suspect since it is clearly "inconvenient" to recognise the Critique without a suitable theoretical response nor the tools to provide a valid practical response.

2. Hendry and Mizon (1996) have expressed a somewhat more general statement as follows; "A model cannot be used for policy if implementing the policy would change the implications from the model, since then the policy outcome would differ from that predicted by the model. A policy which leads to a change in the model parameters which are assumed constant contradicts the basis of itself."

3. See Marcellino and Salmon(2002), Sims (1982a) and Sargent (1984) for further discussion of this view.

4. When viewed as a criticism of estimating "reduced form" models the Critique is really making an argument made several years earlier by Steve Goldfeld and Alan Blinder (1972) when discussing the endogeneity of stabilisation policy and the "St Louis Approach"

5. Under standard conditions, whether in a single player context or the strategic context, if an infinite horizon is maintained, then both players will choose an optimal decision rule that is invariant given the initial formulation of the policy problem . If a finite policy horizon is considered, then a rule which implies continuously changing actions is implied for both players, but this systematically changing policy rule is not that considered in the Lucas Critique since it is perfectly anticipated and accounted for by both players in their formulation of their optimisation problems.

6. This fundamental argument against the Lucas Critique has been put at various times by Sims(1982),(1987),(1988) and Blinder (1984). Sargent (1984) also seems to accept this point but argues from an empirical point of view regarding the optimality of government policy within the sample period consistently with that in the simulation period.

7. LeRoy (1995) refers to this distinction as between stationary and rational expectations. 
8. Notice that a very wide range of values for policy instruments can however follow from a single fixed policy rule with changing input data. Similarly while policy action is obviously intended to alter the dynamic response or properties of the economy this does not imply that the structure of the economy changes.

9. See Hendry and Mizon (1996) for a discussion of super exogeneity and Basar and Olsder (1994) for a discussion of information patterns in dynamic games.

10. Sims (1980, page 12) and (1982, page 110) in fact questions whether in reality government policy is ever sufficiently systematic that a coherent rule could ever be identified.

11. See also Hahn and Solow (1995).

12. In fact we believe the only sensible way to resolve this issue in practice is to formally test the super exogeneity of policy variables using the tools described in Engle and Hendry (1993).

13. Lucas effectively makes this suggestion in section 6 of the 1976 paper but continues to regard a policy change as a parameter shift rather than a new realisation of the stochastic term within the fixed structure of the policy rule.

14. This does not of course mean that policy is random but that uncertainty about policy implies that a stochastic description is feasible. The stochastic specification also captures what Sims means by a regime change which is effectively a policy change which is not captured in the range space of the stochastic policy variable and this is likely to be a very rare event. Which in turn, may make the whole issue of econometric policy evaluation irrelevant if, as mentioned above, such a policy is not captured in the model. Sims' position is then that there may be poor evidence empirically in an existing model if the "input topology" regarding potential policy variation is not rich enough at the time of estimation but this does not necessarily imply that the model is theoretically inadequate as suggested by the Lucas Critique - just poorly estimated. 
15. Similar arguments for robust decision rules can be found in the earlier work of behavioural economists in the 1950's such as Alchian (1950), Baumol and Quandt (1964) and Simon (1959). It turns out that effectively equivalent arguments can also be found in the adjustment cost literature to which Lucas (1967) himself also contributed.

16. Aside from recognising the power of simple integral action, adaptive control theory attempts to estimate the unknown parameters of the system while constructing an optimal decision rule- a method which economists have accepted with the development of theories of learning. However notice that the incorporation of integral action into a decision rule denies the need to learn the unknown parameters perfectly as the decision rule robustly takes up the slack created by imperfect knowledge of the constraints. For further discussion see Salmon ((1993).

17. For the present the target is taken as a constant but the theory generalises to more general dynamic forms for $y^{*}$ with different costs of adjustment.

18. Notice also that the wide use of error or equilibrium correction mechanisms in the specification large macroeconometric models would seem to have been empirically justified and is one clear difference between present day macroeconometric models and their predecessors. 


\section{References:}

Athans M,(1971), On the design of PID controllers using optimal linear regulator theory, Automatica,7,643-647.

Alchian A., (1950),Uncertainty , evolution and economic theory, Jnl. of Political Economy, 58, pp. 211-21.

Bạsar T. and Bernhard P. (1995) $H^{\infty}$ - Optimal Control and Related MinMax Design Problems; a Dynamic Game Approach, 2nd edition Birkhäuser.

Bạsar T. and Olsder G. (1995) Dynamic Noncooperative Game Theory, 2nd. Edition, Academic Press, London.

Baumol W.J. and Quandt R.E. (1964), Rules of thumb and optimally imperfect decision rules, American Economic Review, 54, pp. 23-46.

Bernhard P.,(2002), A Survey of Linear Quadratic Robust Control, (this volume)

Blinder A. (1984),discussion of T.Sargent Vector autoregressions, expectations, and advice, American Economic Review, 74, pp.417-419.

Cooley T.F.,Le Roy S.F. and Raymon N. (1984), Econometric policy evaluation: a note, American Economic Review, 74, no.3 pp. 467-470.

Dupuis P.,James M., and Petersen I., (2000), Robust Properties of RiskSensitive Control, Mathematics of Control, Signals and Systems,13,318332 .

Engle, R.F. and Hendry D.F. (1993), Superexogeneity and invariance in regression models, Journal of Econometrics, 56, pp. 119-139.

Epstein L.G. and Zin S.E.,Substitution, Risk Aversion and the Temporal Behaviour of Consumption and Asset Returns; A Theoretical Framework, Econometrica, 57,4,937-969.

Ericsson, N.R. and Irons J.S. (1996), The Lucas Critique in practice: theory without measurement, pages 263-312 in Hoover K. (ed), Macroeconometrics: Developments, Tensions and Prospects ,Kluwer Academic Press, Dordrecht. 
Favero, C. and Hendry D.F. (1992), Testing the Lucas Critique: a review, Econometric Reviews, 11, pp. 265-306.

Francis B.A. and Wonham W.M. (1976), The internal model principle of control theory, Automatica, 12,pps 457-465.

Gilboa I. and D. Schmeidler,(1989), Maxmin Expected Utility with Nonunique prior, Journal of Mathematical Economics, 18,141-153.

Goldfeld S.M. and Blinder A.S. (1972), Some implications of endogenous stabilization policy, Brookings Papers on Economic Activity, 3, pp. 585640.

Green M. and D. Limebeer,(1995), Linear Robust Control, Prentice- Hall , Englewood Cliffs,NJ.

Hahn F. and Solow R. (1995) A Critical Essay On Modern Macroeconomic Theory, MIT Press.

Hansen L.P. and Sargent T.J. (1980), Formulating and Estimating Dynamic Linear Rational Expectations Models, Journal of Economic Dynamics and Control, 2, pp. 7-46.

Hansen, L.P. and Sargent T.J. (1981), Formulating and estimating dynamic linear rational expectations models, in Lucas, R.E. and Sargent T.J. (1981).

Hansen, L.P. and Sargent T.J. (1992), Discounted linear exponential quadratic gaussian control, unpublished manuscript, University of Chicago.

Hansen L.P. and Sargent T.J.,(1999), Elements of Robust Control and Filtering in Macroeconomics, mimeo.

Hansen L.P. Sargent T.J. and Tallerini T.(1999),Robust Permanent Income and Pricing, Review of Economic Studies.66,873-90\%.

Hendry D.F. (1995), Dynamic Econometrics,Oxford University Press, Oxford.

Hendry, D.F.and Anderson G. (1977), Testing Dynamic Specification in Small Simultaneous Systems: an Application to a Model of Building Society Behaviour in the UK, in M. Intrilligator (ed.),Frontiers in Quantitative Economics,North Holland. 
Hendry, D.F.and Mizon G.E. (1996), The Role of Exogeneity in Economic Policy Analysis, Journal of Business and Economic Statistics .

Hendry D.F. and von Ungern-Sternberg (1981), Liquidity and inflation effects on consumers' expenditure, in Deaton A.S. (ed.),Essays in the Theory and Measurement of Consumers' Behaviour,pp 237-261, Cambridge : Cambridge University Press .

Hoover, K.D. (1995), Macroeconometrics: Developments, Tensions and Prospects,Kluwer Academic Press, Dordrecht.

Kamien, M.I. and Schwartz N.L. (1981), Dynamic Optimization, North Holland.

Kirman, A. (1992), Whom or What Does the Representative Individual Represent?, Journal of Economic Perspective, 6, pp. 117-136.

Knight F.H. (1921), Risk, Uncertainty and Profit, Houghton Muffin, Boston.

LeRoy, S.F. (1995), On policy regimes, pages 235-251 in Hoover K. (ed), Macroeconometrics: Developments, Tensions and Prospects ,Kluwer Academic Press, Dordrecht.

Lucas R.E. (1967), Adjustment costs and the theory of supply, Journal of Political Economy, vol 75.

Lucas, R.E. (1976), Econometric Policy Evaluation: A Critique, in K. Brunner and A.H. Meltzer (eds.), The Phillips Curve and Labour Markets, Amsterdam: North Holland, pp. 19-46.

Lucas, R.E. and Sargent T.J. (1981), Introduction toRational Expectations and Econometric Practice, Lucas, R.E. and Sargent T.J. (1981).

Lucas, R.E. and Sargent T.J. (eds.), (1981), Rational Expectations and Econometric Practice, University of Minnesota Press.

Marcellino M. (1995), A further comment on econometric policy evaluation, mimeo European University Institute.

Marcellino M. and Salmon M.,(2002), On the theoretical irrelevance of the Lucas Critique, twenty five years on, in M. Salmon(ed), Robust and Risk Sensitive Decision Theory in Economics and Finance, forthcoming Cambridge University Press. 
Miller, M. and Salmon M. (1985a), Policy coordination and the time inconsistency of optimal policy in an open economy, Economic Journal, Suppl., pp. 124-135.

Miller, M. and Salmon M. (1985b) Dynamic games and time consistent policy in open economies, in International Policy Co-ordination, NBER/CEPR edited by W. Buiter and R. Marston, CUP, pp 184-213,.

Nickell S.J. (1985), Error correction, partial adjustment and all that: An expository note, Oxford Bulletin of Economics and Statistics, 47, pp.119130.

Onatski and J. Stock, (2002), Robust Monetary Policy Under Model Uncertainty in a small Model of the U.S. Economy.,this volume.

Petersen Ian, V.Ugrinovskii and A. Savkin,(2001), Robust Control Design using $H^{\infty}$ Methods, Springer .

Phillips A.W. (1954), Stabilisation Policy in the Closed Economy, The Economic Journal,67, pps 290-323.

Rosenbrock H.H. and McMorran (1971), Good, bad or optimal ,I.E.E.E. Transactions on Automatic Control,Vol 16, no.6.

Salmon M. (1981), Econometric implications of time inconsistency, mimeo University of Warwick.

Salmon M. (1982), Error correction mechanisms, The Economic Journal,92, pp 615-629.

Salmon M. (1984), Some notes on modelling optimising behaviour in the absence of an optimal theory, mimeo, paper prepared for H.M.Treasury Academic Panel Meeting.

Salmon M. (1988), Error correction models, cointegration and the internal model principle, Journal of Economic Dynamics and Control,12, pp.523-549.

Salmon M. (1993), Bounded rationality and learning; procedural learning, in Learning and Rationality in Economics, Kirman A. and Salmon M. (eds.), Basil Blackwell. 
Salmon M. and Young P. (1978), Control methods and quantitative economic policy, in Optimal Control for Econometric Models; An Approach to Economic Policy Formulation, S.Holly, Rustem B. and Zarrop M. (eds), MacMillan

Sargent, T.J. (1981), Interpreting economic time series,Jnl. of Political Economy,89, pp 213-248.

Sargent, T.J. (1984), Vector autoregressions, expectations, and advice,American Economic Review, 74, pp.408-415.

Simon H., C.A. (1959), Theories of decision making in economics and behavioural science, American Economic Review, 49(1), pp. 253-83.

Sims, C.A. (1980), Macroeconomics and reality, Econometrica, 48, pp. 1-48.

Sims, C.A. (1982a), Policy analysis with econometric models, Brookings Papers on Economic Activity, 1, pp. 107-164.

Sims, C.A. (1982b), 'Scientific standards in econometric modellingin Current Developments in the Interface: Economics, Econometrics, Mathematics,edited by M.Hazewinkel and A.H.G. Rinnoy Kan, 317-37. Dordrecht, Boston, London, Reidel.

Sims, C.A. (1986), Are forecasting models useful for policy analysis?, Quarterly Review of the Federal Reserve Bank of Minneapolis, Winter, 2-16.

Sims C. (1987), A rational expectations framework for short run policy analysis, Chapter 14 in New Approaches to Monetary Economics, W.A.Barnett and K.J. Singleton (eds.).

Sims C. (1988), Identifying policy effects, in Empirical Macroeconomics for Interdependent Economies,edited by Ralph Bryant et al., Washington, Brookings Institution.

Whittle P.,(1990), Risk Sensitive Optimal Control, New York, Wiley.

Whittle P.,(1996),Optimal Control: Basics and Beyond, New York, Wiley.

Whittle P.(2002), Risk Sensitivity- A Strangely Pervasive Concept, this volume. 
Zhou K., J.C. Doyle and K.Glover (1996), Robust and Optimal Control,PrenticeHall. 


\section{List of other working papers:}

\section{1}

1. Soosung Hwang and Steve Satchell, GARCH Model with Cross-sectional Volatility; GARCHX Models, WP01-16

2. Soosung Hwang and Steve Satchell, Tracking Error: Ex-Ante versus Ex-Post Measures, WP01-15

3. Soosung Hwang and Steve Satchell, The Asset Allocation Decision in a Loss Aversion World, WP01-14

4. Soosung Hwang and Mark Salmon, An Analysis of Performance Measures Using Copulae, WP01-13

5. Soosung Hwang and Mark Salmon, A New Measure of Herding and Empirical Evidence, WP01-12

6. Richard Lewin and Steve Satchell, The Derivation of New Model of Equity Duration, WP0111

7. Massimiliano Marcellino and Mark Salmon, Robust Decision Theory and the Lucas Critique, WP01-10

8. Jerry Coakley, Ana-Maria Fuertes and Maria-Teresa Perez, Numerical Issues in Threshold Autoregressive Modelling of Time Series, WP01-09

9. Jerry Coakley, Ana-Maria Fuertes and Ron Smith, Small Sample Properties of Panel Timeseries Estimators with I(1) Errors, WP01-08

10. Jerry Coakley and Ana-Maria Fuertes, The Felsdtein-Horioka Puzzle is Not as Bad as You Think, WP01-07

11. Jerry Coakley and Ana-Maria Fuertes, Rethinking the Forward Premium Puzzle in a Nonlinear Framework, WP01-06

12. George Christodoulakis, Co-Volatility and Correlation Clustering: A Multivariate Correlated ARCH Framework, WP01-05

13. Frank Critchley, Paul Marriott and Mark Salmon, On Preferred Point Geometry in Statistics, WP01-04

14. Eric Bouyé and Nicolas Gaussel and Mark Salmon, Investigating Dynamic Dependence Using Copulae, WP01-03

15. Eric Bouyé, Multivariate Extremes at Work for Portfolio Risk Measurement, WP01-02

16. Erick Bouyé, Vado Durrleman, Ashkan Nikeghbali, Gael Riboulet and Thierry Roncalli, Copulas: an Open Field for Risk Management, WP01-01

\section{0}

1. Soosung Hwang and Steve Satchell, Valuing Information Using Utility Functions, WP00-06

2. Soosung Hwang, Properties of Cross-sectional Volatility, WP00-05

3. Soosung Hwang and Steve Satchell, Calculating the Miss-specification in Beta from Using a Proxy for the Market Portfolio, WP00-04

4. Laun Middleton and Stephen Satchell, Deriving the APT when the Number of Factors is Unknown, WP00-03

5. George A. Christodoulakis and Steve Satchell, Evolving Systems of Financial Returns: AutoRegressive Conditional Beta, WP00-02

6. Christian S. Pedersen and Stephen Satchell, Evaluating the Performance of Nearest Neighbour Algorithms when Forecasting US Industry Returns, WP00-01

1999

1. Yin-Wong Cheung, Menzie Chinn and Ian Marsh, How do UK-Based Foreign Exchange Dealers Think Their Market Operates?, WP99-21 
2. Soosung Hwang, John Knight and Stephen Satchell, Forecasting Volatility using LINEX Loss Functions, WP99-20

3. Soosung Hwang and Steve Satchell, Improved Testing for the Efficiency of Asset Pricing Theories in Linear Factor Models, WP99-19

4. Soosung Hwang and Stephen Satchell, The Disappearance of Style in the US Equity Market, WP99-18

5. Soosung Hwang and Stephen Satchell, Modelling Emerging Market Risk Premia Using Higher Moments, WP99-17

6. Soosung Hwang and Stephen Satchell, Market Risk and the Concept of Fundamental Volatility: Measuring Volatility Across Asset and Derivative Markets and Testing for the Impact of Derivatives Markets on Financial Markets, WP99-16

7. Soosung Hwang, The Effects of Systematic Sampling and Temporal Aggregation on Discrete Time Long Memory Processes and their Finite Sample Properties, WP99-15

8. Ronald MacDonald and Ian Marsh, Currency Spillovers and Tri-Polarity: a Simultaneous Model of the US Dollar, German Mark and Japanese Yen, WP99-14

9. Robert Hillman, Forecasting Inflation with a Non-linear Output Gap Model, WP99-13

10. Robert Hillman and Mark Salmon , From Market Micro-structure to Macro Fundamentals: is there Predictability in the Dollar-Deutsche Mark Exchange Rate?, WP99-12

11. Renzo Avesani, Giampiero Gallo and Mark Salmon, On the Evolution of Credibility and Flexible Exchange Rate Target Zones, WP99-11

12. Paul Marriott and Mark Salmon, An Introduction to Differential Geometry in Econometrics, WP99-10

13. Mark Dixon, Anthony Ledford and Paul Marriott, Finite Sample Inference for Extreme Value Distributions, WP99-09

14. Ian Marsh and David Power, A Panel-Based Investigation into the Relationship Between Stock Prices and Dividends, WP99-08

15. Ian Marsh, An Analysis of the Performance of European Foreign Exchange Forecasters, WP99-07

16. Frank Critchley, Paul Marriott and Mark Salmon, An Elementary Account of Amari's Expected Geometry, WP99-06

17. Demos Tambakis and Anne-Sophie Van Royen, Bootstrap Predictability of Daily Exchange Rates in ARMA Models, WP99-05

18. Christopher Neely and Paul Weller, Technical Analysis and Central Bank Intervention, WP9904

19. Christopher Neely and Paul Weller, Predictability in International Asset Returns: A Reexamination, WP99-03

20. Christopher Neely and Paul Weller, Intraday Technical Trading in the Foreign Exchange Market, WP99-02

21. Anthony Hall, Soosung Hwang and Stephen Satchell, Using Bayesian Variable Selection Methods to Choose Style Factors in Global Stock Return Models, WP99-01

\section{8}

1. Soosung Hwang and Stephen Satchell, Implied Volatility Forecasting: A Compaison of Different Procedures Including Fractionally Integrated Models with Applications to UK Equity Options, WP98-05

2. Roy Batchelor and David Peel, Rationality Testing under Asymmetric Loss, WP98-04

3. Roy Batchelor, Forecasting T-Bill Yields: Accuracy versus Profitability, WP98-03

4. Adam Kurpiel and Thierry Roncalli, Option Hedging with Stochastic Volatility, WP98-02

5. Adam Kurpiel and Thierry Roncalli, Hopscotch Methods for Two State Financial Models, WP98-01 